THE Astrophysical Journal SuPPLEment Series, 90:515-520, 1994 February

(c) 1994. The American Astronomical Society. All rights reserved. Printed in U.S.A.

\title{
PARTICLE ACCELERATION MECHANISMS
}

\author{
R. D. BLANDFORD \\ Theoretical Astrophysics 130-33, California Institute of Technology, Pasadena, CA 91125 \\ Received 1993 March 16; accepted 1993 June 9
}

\begin{abstract}
High-energy particle acceleration is observed to proceed in a diverse variety of astrophysical sites ranging from the terrestrial aurorae to the most distant quasars. Particle acceleration is a fairly common channel for the release of large-scale kinetic, rotational, and magnetic energy. Physical mechanisms include electrostatic acceleration, stochastic processes and diffusive shock energization. Cosmic-ray energy spectra have shapes which reflect escape, collisional, and radiative losses. The overall acceleration efficiency is controlled by the low-energy particle injection which may, in turn, feed back into the energization. Recent observational developments, which illustrate these general principles and raise fresh questions, are briefly summarized.
\end{abstract}

Subject heading: acceleration of particles

\section{SITES OF PARTICLE ACCELERATION}

The selection and acceleration of a small minority of charged particles out of a cosmic plasma occurs in a diverse variety of sites throughout the universe. In Figure 1, we exhibit a schematic plot of the characteristic distances from Earth $D$ against the highest particle energy, $E$, for which we have reasonable evidence in a variety of acceleration sites. (In order to accommodate the large dynamic range in both of these quantities, I plot the logarithms of their logarithms!) This plot is, naturally, not comprehensive. Translations of the acronyms are as follows:

TPA-Terrestrial particle accelerators. $\sim \mathrm{TeV}$ at present, projected up to $\sim 20 \mathrm{TeV}$ for the SSC. In the past, space physics has been the beneficiary of laboratory experience. As the cost of conventional accelerators appears to diverge with energy, innovative ideas drawn from cosmic and laboratory plasma physics may become important (e.g., Dawson 1989).

AUR-Terrestrial aurorae. Typically, $\mathrm{keV}$ energies are involved and electric field components parallel to the magnetic field are involved in the acceleration (e.g., Evans 1987).

MAG-Terrestrial bow shock and magnetosphere. Particles are accelerated to energies as high as $\sim 100 \mathrm{MeV}$ within the inner magnetosphere and to somewhat lower energies at both the bow shock and the magnetotail (e.g., Parks 1991).

JSUN-Outer planets. Similar particle acceleration has been observed by Pioneer, Voyager, and Ulysses in the outer planets (e.g., Van Allen 1987).

IPM-Interplanetary medium. A variety of interplanetary shock waves (e.g., corotation interaction regions, co-rotating ion events, termination shock ) accelerate particles that are observed in situ (e.g., Lee 1992).

SOL-Solar flares. These are famous accelerators of electrons and ions and emitters of neutrons, $\gamma$-rays, and radio waves (e.g., Melrose 1992).

STAR-Stars. Nearby stars are also prone to flaring, and in some cases, are much more active than the Sun as evidenced by radio and X-ray observations (e.g., Bastian et al. 1990).

XRB-Binary X-ray sources. These must also be capable of accelerating electrons at least to $\sim \mathrm{MeV}$ energies. The most direct evidence comes from observations of electron-positron annihilation lines in black-hole candidate X-ray novae (e.g., Sunyaev 1992). Several detections of binary X-ray sources have been reported at Tev and even PeV energies. However, in view of the confusion in this field, I shall ignore these for the moment (e.g., Ruderman 1991).

GC-Galactic center. The true dynamical center appears to be coincident with the radio source SgrA*, and this has recently been detected as a $\gamma$-ray source (Mattox 1993).

PSR - Radio pulsars. The observation of $\mathrm{TeV} \gamma$-rays from the Crab Nebula by the Whipple Observatory indicates that electrons and positrons, of even larger energy, must be accelerated, presumably at the pulsar wind termination shock (e.g., Arons 1992).

SNR-Supernova remnants. We only have direct evidence for the acceleration of $\sim 10 \mathrm{GeV}$ electrons. However, it is widely believed that Galactic cosmic rays with energy $\$ 10^{6}$ $\mathrm{GeV}$ are accelerated by the bounding shock waves (e.g., Axford 1992).

QSO-Radio quiet quasars (and Seyfert galaxies). These have been observed by OSSE and COMPTEL and appear to cut off at $\sim 100 \mathrm{keV}$ (e.g., Cameron et al. 1993).

CRS-Compact, radio-loud AGNs. EGRET observations exhibit a high detection rate among "Blazars" (the union of the so-called BL Lac objects and "optically violently variable" quasars). $\gamma$-rays up to $10 \mathrm{GeV}$ in energy have been observed and some sources appear to vary on timescales of days (Fichtel et al. 1993). In one case, Mrk 421, $\sim 1 \mathrm{TeV} \gamma$-rays have been reported (Punch et al. 1992).

ERS-Extended radio sources. The large $\gg 1 \mathrm{kpc}$ components of double radio sources are the sites for acceleration of $\sim 10 \mathrm{GeV}$ relativistic electrons responsible for radio synchrotron emission. They may also be the sources of he most energetic cosmic rays (with energies $\sim 10^{20} \mathrm{eV}$ ) observed at Earth (e.g., Hillas 1984).

GRB $-\gamma$-ray bursters. The brightest sources radiate most of their power in the $\sim 100 \mathrm{keV}-1 \mathrm{MeV}$ range and have been observed at energies $\gtrsim 100 \mathrm{MeV}$ (e.g., Fishman 1993). Recent BATSE observations have confirmed that the sources are distributed isotopically but have also exhibited a deficit of faint 


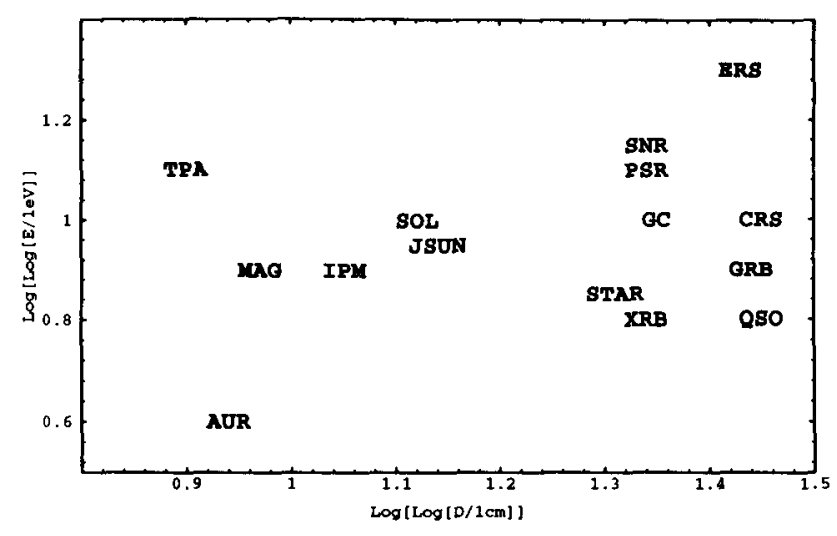

Fig. 1.-Particle acceleration sites characterized by their distances from Earth, $D$, and the maximum energy to which particles can be accelerated. The acronyms are translated in the text.

bursts so that we must be seeing to the edge of their radial distribution. They have been associated with sources as close as the Oort cloud and as distant as high-redshift galaxies (Meegan et al. 1992).

\section{SOURCES OF FREE ENERGY}

I have been asked to look at the problem of particle acceleration from a physical standpoint. It is obvious from Figure 1 that no single particle acceleration mechanism can be at work. Conversely, and perhaps encouragingly, theoretical particle acceleration mechanisms, that are physically self-consistent, may operate in sites quite different from those for which they were originally proposed and this possibility provides the main justification for a cross-disciplinary meeting like this.

The first requirement on a theory of particle acceleration is a source of free energy. Of course, in a typical particle acceleration site, energy changes form many times and there is some ambiguity in defining the "source." I shall, take this word to refer to that agency whose energy is permanently decreased in order to power the acceleration. Three types of sources are commonly invoked.

\subsection{Bulk Kinetic Energy of Fluid Motion}

The most common source is bulk kinetic energy, usually in the form of a shock front. This is often the consequence of an explosion, like a supernova or a coronal mass ejection responsible for a major proton solar flare. In the former case, the energy is ultimately gravitational and in the latter presumably drawn from the slow, convective motion below the solar photosphere. However, the particle acceleration is quite ignorant of this lineage. Shock acceleration can also occur at standing shock fronts, notably planetary bow shocks that dissipate the kinetic energy flux in the solar wind [typically, $\sim 0.1(r / 1 \mathrm{AU})^{-2} \mathrm{ergs}$ $\mathrm{s}^{-1} \mathrm{~cm}^{-2}$ ]. It is interesting that the energy fluxes and Mach numbers in the outer solar wind are not so different from those encountered in old supernova remnants. (What is markedly different between these two sites, however, are the time and length scales which ultimately limit the energy to which particles are accelerated.)

A different type of shock wave that has been studied more intensively in recent times is a relativistic shock. This may also take the form of a standing shock, as in the termination shock of the Crab pulsar wind, or may travel at ultrarelativistic speed, as in the compact jets of extragalactic radio sources.

\subsection{Rotational Energy}

A second, generic energy source is rotation. This is most usually tapped for particle acceleration through some version of unipolar induction. This has been invoked in the Jupiter-Io system, radio pulsars, and, more speculatively, black holes in both active galactic nuclei and stellar binary systems.

\subsection{Magnetic Energy}

Finally, we have the energy released when some magnetostatic configuration is slowly stressed to the point of dynamical instability. The difference in magnetic energy between the initial and final configuration appears ultimately, mostly as heat. However, if the energy is released sufficiently rapidly, a strong shock can be found, as above. Alternately, and often simultaneously, energy is dissipated in the vicinity of a neutral point or line. This happens in Earth's magnetotail and in impulsive solar flares. It is also conjectured to occur at the Alfvén radius where a neutron star magnetosphere interacts with accreting gas and within the coronal regions above accretion disks.

A common characteristic of astrophysical particle acceleration is that it is very efficient with over $10 \%$ of the available energy being channelled into high-energy particles. This is a serious constraint on particle acceleration mechanisms.

\section{GENERIC ACCELERATION MECHANISMS}

\subsection{Direct Electrostatic Acceleration}

Charged particles change their energies through the application of electric field; magnetic fields are "lazy" and do no work. This happens most directly under electrostatic conditions. Under most circumstances, though, plasma cannot sustain electric fields for lengths in excess of a Debye length or times in excess of a plasma period. However, when a conductor moves in a magnetic field, a permanent potential difference is created and this is available for particle acceleration. The principle is most simply illustrated using an axisymmetric, unipolar inductor (e.g., Goldreich \& Julian 1969).

Consider a stationary, axisymmetric, conducting star or planet rotating with angular velocity $\Omega$ and magnetized parallel to its spin. In the inertial, nonrotating frame, $E=-(\Omega \times r) \times$ $B / c$ and the surface of the conductor will, therefore, have a potential gradient across it. As the magnetic field is constant, the total emf around any circuit is zero and so this same potential difference must also be maintained across the magnetosphere. Strong magnetic field lines essentially act as wires (moving with the speed of the $\boldsymbol{E} \times \boldsymbol{B}$ drift and a current will flow of strength given by the magnetospheric resistance. (The internal resistance of the star battery is generally small.) When the field lines and the circuit extend to infinity under electromagnetic conditions, as happened around a radio pulsar or a magnetized black hole, the effective impedance is roughly that of free space or $Z_{0} \sim 100 \mathrm{Ohm}$. If the magnetic flux threatening the conductor is $\Phi$, then the voltage is $V \sim \Omega \Phi / c$ to order of magnitude. The associated current is $V / Z_{0}$ and the power that is carried away from the battery in the form of an electromagnetic Poynting flux is $P \sim V^{2} / Z_{0}$. For example, in the case of 
the Crab pulsar, $P \sim 10^{31} \mathrm{~W}$ so that $V \sim 3 \times 10^{16} V, I \sim 3 \times$ $10^{14} \AA$ and the surface magnetic field strength is $B \sim 10^{12} \mathrm{G}$. The current flows across the magnetic field inside the neutron star exerting a torque. Where it flows through the magnetosphere, and beyond, depends upon the microscopic details of the plasma physics.

In many contemporary pulsar models, "outer gaps" are envisaged to open up within the magnetosphere, across which some fraction of the total rotational potential difference (typically $\sim 10^{12} \mathrm{~V}$ ) is sustained (e.g., Arons 1992). TeV electrons can be accelerated across these gaps, and these particles can subsequently produce electron-positron secondaries through pair creation on the virtual photons associated with the magnetostatic field. This relativistic, electron-positron plasma is believed to emit coherent radio waves. The observation of pulsed $\gamma$-rays from the Crab pulsar with a power that is a significant fraction of the total spin-down luminosity, suggests that at least some of the circuit can be closed within the magnetosphere. Other possible sites for current closure include the vicinity of the light cylinder at $r=c / \Omega$, the pulsar wind termination shock at $r \sim 10^{17} \mathrm{~cm}$ (where the synchrotron-emitting relativistic electrons are accelerated) and the edge of the pulsar wind cavity at $\sim 10^{18} \mathrm{~cm}$. If high-energy particles accelerated at the outer shock in the interstellar medium are able to leak into the nebula, then they can accelerate to energies as large as $V$ by drifting between the pole and the equator. This has been proposed as an acceleration mechanism for Galactic cosmic rays in the $\sim 10^{14}-10^{18} \mathrm{eV}$ energy range (Bell 1992).

A more controversial example of electrostatic acceleration is given by double layers. These are apparently observed with potential differences $\sim 1 \mathrm{~V}$ above auroral zones. It has been postulated that strong double layers supporting potential differences orders of magnitude in excess of $k T / e$ form readily when plasmas carry currents (e.g., Kuijpers 1990).

Magnetic reconnection is another source of inductive electric field. Under the Petschek model, the flow is stationary and there is a motional electric field of $E \sim 0.1 a B / c$ where $a$ is the Alfvén speed. However, in Earth's magnetotail (and elsewhere) the situation is envisioned to be more complicated. Rapid reconnection in a reversed field region is supposed to occur and in the nonlinear phase, the magnetic perturbation can grow explosively, $\delta B \propto(\tau-t)^{-1}$ for some time $\tau$, and this allows particles to be accelerated to energies $\sim 1 \mathrm{MeV}$, in excess of the total motional potential difference across Earth's magnetosphere (e.g., Galeev 1991). A major difficulty that must be overcome if this acceleration mechanism is to apply on larger scales is that the efficiency for acceleration to high energies is likely to be quite low as very few particles actually encounter the neutral line.

\subsection{Stochastic Acceleration}

Instead of particles gaining energy in one giant step, some acceleration mechanisms postulate that wave turbulence causes particles to diffuse through momentum space in many small steps $\Delta p$. If the time interval between these steps is denoted $\Delta t$, we can define a diffusion coefficient

$$
D_{p p}=\left\langle\frac{\Delta p \Delta p}{\Delta t}\right\rangle
$$

and the momentum space distribution function $f(\boldsymbol{p}, \boldsymbol{x}, \boldsymbol{t})$ satisfies a specialized form of the Fokker-Planck equation

$$
\frac{\partial f}{\partial t}=\nabla_{p} \cdot D_{p p} \nabla_{p} f
$$

(e.g., Achterberg 1992). (Additional terms may be added to the right-hand side of this equation corresponding to loss and escape.)

In one simple example of stochastic acceleration, Alfvén waves interact resonantly with particles whose Larmor radii, $r_{\mathrm{L}}$ match their wavelength. Now, if we think of Alfvén waves as quanta with momenta $\hbar \boldsymbol{k}$ and energy $\hbar \omega$, then the ratio of their energies to their momenta $\sim a$ is much less than the corresponding ratio for high-energy particles, which is $\sim c$. Alfvén waves are therefore usually most efficient at adding momentum in steps $\Delta \boldsymbol{p}$ almost perpendicular to $\boldsymbol{p}$, that is to say Alfvén waves are more likely to be involved in scattering than directly accelerating particles. The scattering rate is usually expressed as a diffusion coefficient in the pitch angle $\phi$,

$$
D_{\phi \phi} \equiv \nu \equiv\left(\frac{\Delta \phi^{2}}{\Delta t}\right) \sim\left(\frac{\delta B}{B}\right)_{k \sim r_{\bar{L}}^{-1}}^{2}\left(\frac{c}{r_{\mathrm{L}}}\right) .
$$

$(\delta B / B)$ is the relative amplitude of the resonant Alfvén waves. Associated with the scattering is a spatial diffusion coefficient:

$$
D_{E E} \sim E^{2}\left(\frac{a}{v}\right) \omega\left(\frac{\delta B}{B}\right)^{2}
$$

Magnetosonic waves are more effective at accelerating particles. Here the fundamental resonance is that associated with Landau damping, $\omega=k_{\|} v_{\|}$, where the subscript \| indicates projection along the magnetostatic field and $\omega$ is magnetosonic wave frequency. In this case, the energy diffusion coefficient becomes

$$
D_{E E} \sim E^{2}\left(\frac{a}{v}\right) \omega\left(\frac{\delta B}{B}\right)^{2}
$$

This type of acceleration can also occur in nonlinear Landau damping of the beat wave formed by two Alfvén waves. Note that for relativistic particles, the rate of gain of energy is proportional to the energy as in a traditional Fermi process. In order to produce a power-law distribution function requires this prescription for acceleration to be combined with an exponential distribution of acceleration times. Processes like this might be responsible for creating the seed particles for more efficient acceleration processes.

A general concern about stochastic acceleration by wave turbulence is that often the observed wave intensities are inferred, or in the case of the interplanetary medium, observed to be so large that the quasi-linear approach underlying the FokkerPlanck formalism is of questionable validity.

\subsection{Diffusive Shock Acceleration}

A somewhat different physical approach must be followed to analyze diffusive shock acceleration. Here the particle energies are derived from the relative motion between scatterers on either side of a strong shock wave. As the scattering is supposed 
strong, we need only deal with the isotropic part of the particle distribution function. This will be convected by the flow with velocity $\boldsymbol{u}(\boldsymbol{x})$ and will diffuse down a concentration gradient. There is a second physical effect, the acceleration of particles in a converging flow, which must be included. The net result is the convection diffusion equation which, in its simplest form can be written in one dimension as

$$
u \frac{\partial f}{\partial x}-\frac{\partial}{\partial x} D_{x x} \frac{\partial f}{\partial x}=\frac{1}{3} \frac{d u}{d x} \frac{\partial f}{\partial \ln p}
$$

(e.g., Jones \& Ellison 1991). Now suppose that the shock is treated as a mathematical discontinuity where the density increases by a factor $r$. If the particle distribution function asymptotically upstream is denoted $f_{-}\left(p^{\prime}\right)$, then the transmitted distribution asymptotically downstream can be shown to be

$$
f_{+}(p)=q p^{-q} \int_{0}^{p} d p^{\prime} f\left(p^{\prime}\right) p^{q-1}
$$

where $q=3 r /(r-1)=4 M^{2} /\left(M^{2}-1\right)$ for a high $\beta$ shock with Mach number $M$.

This result implies that relativistic particles will be transmitted with a power-law distribution function, $d N / d E \tilde{\propto} E^{-2}$ by high Mach number shocks as observations of Galactic cosmic rays and radio supernova remnants roughly require (see below). Naturally, there are many genuine complications which have stimulated many equally complicated analyses. These include the back reaction of the accelerated particles on the flow which, in turn, modifies the velocity field and the transmitted spectrum (e.g., Reynolds \& Ellison 1993) and the influence of the magnetic stress field on the shock dynamics.

Shocks are usually subdivided into quasi-parallel shocks (when the magnetic field is roughly perpendicular to the shock front) and quasi-perpendicular shocks (when the field is roughly parallel). When the fluid velocity is not strictly parallel to the magnetic field, there is a motional electric field and its action upon the charged particles is sometimes thought to be responsible for particle acceleration. This process is known as shock drift acceleration. In simple cases, shock drift acceleration can be shown to be unimportant because it is possible to make a Lorentz transformation parallel to the shock surface into a frame where the fluid velocity $\boldsymbol{u}(x)$ is parallel to $\boldsymbol{B}$ so that there is no motional electric field. (This is known as a "de Hoffman-Teller" transformation.) However, such a global transformation is not possible when the shock is nearly perpendicular or when the shock surface is curved. Some authors believe that shock drift acceleration dominates the acceleration.

An issue of great concern is what is the maximum energy to which a particle can be accelerated by the diffusive shock process? This is fixed by equating the diffusive scale length ahead of the shock to its radius of curvature. If we make the (strong) assumption that the scattering waves are all of nonlinear strength, then it turns out that the limiting energy is typically of order the motional potential difference across the shock (times the particle charge). This evaluated to $\sim 10^{14} \mathrm{eV}$ for Galactic supernova remnants.
A recent development has been the numerical simulation of ultrarelativistic perpendicular shocks. In a particular simulation that may be appropriate for the Crab pulsar termination shock, a particle-dominated, wind moving with bulk Lorentz factor $\sim 10^{6}$ passes through a strictly perpendicular shock (Arons et al. 1994). What is reported to happen next depends upon whether the momentum of the positive charges is carried mostly by positrons or protons. In the former case, the downstream particle distribution has roughly a relativistic Maxwellian form, whereas in the latter, the ions make large amplitude cyclotron waves which are absorbed by the electrons to transmit a distribution function again with power-law form $N_{E} \propto$ $E^{-2}$. This neatly accounts for the optical- $\gamma$-ray continuum emission from the Crab nebula. Two residual concerns about this calculation are the sensitivity to the assumption that the shock is strictly perpendicular and the dependence upon the artificially large electron-proton mass ratio which was used for computational reasons.

\section{COMPLICATIONS}

Given a feasible particle acceleration process, there are several more effects which must be taken into account in order to relate theory to observation.

\subsection{Losses}

Shock waves are expanding flows and even if diffusive shock acceleration is efficient, the energy taken up by the relativistic particles will be partially lost in the subsequent adiabatic decompression. Typically, if the characteristic scale size of the region is $L(t)$, then, individual particle momenta scale $p \propto$ $L^{-1}$ in accordance with DeBroglie's principle. This is important in giant solar flares and supernova remnants.

Radiative losses are particularly important in active galactic nuclei. If the combined magnetic and soft photon energy density is denoted by $U$, then a relativistic electron of energy $\gamma m c^{2}$ cools in a time $\sim 2 \times 10^{7}\left(U / 1 \text { ergs } \mathrm{cm}^{-3}\right)^{-1} \gamma^{-1} \mathrm{~s}$. This implies that steady acceleration processes like diffusive shock acceleration may not be able to account for high energy electrons. This restriction does not, of course, apply to ions.

Ionization loss, where the cooling time is $\sim 7 E_{\mathrm{kev}}^{3 / 2} Z^{-2}$ years for nonrelativistic ions of charge $Z$ is catastrophic at low energy and would, in any case, produce a quite foreign abundance distribution. This implies that rapid preacceleration or injection mechanisms are at work.

\subsection{Secondary Particles}

Losses can also be turned to advantages. If, for example, protons but not electrons are accelerated to high energy in AGNs, the relativistic electrons and $\gamma$-rays may still be created as secondaries. Collisions with stationary protons create charged pions, which ultimately decay into electrons and positrons and neutral pions, which produce $\gamma$-rays (e.g., Begelman, Rudak, \& Sikora 1990). Spallation reactions on heavier nuclei release neutrons which may propagate large distances before decaying into electrons. This also happens in solar flares where the neutrons are directly observed. These processes are likely to dominate below $\sim 10^{4} \mathrm{GeV}$. Ultra-high-energy protons in 
AGNs will be decelerated mainly through photon interactions. Doppler shifting a soft X-ray photon into the rest frame of the relativistic proton produces an energetic $\gamma$-ray which may pair produce on the proton's Couloumb field (an even convert target protons into neutrons).

Similar processes are known to occur in the interstellar medium and essentially all of the light $\mathrm{Li}, \mathrm{Be}$, and $\mathrm{B}$ cosmic rays are made in this manner. As roughly half the $\sim 5 \mathrm{GeV}$ medium cosmic rays (e.g., $\mathrm{C}, \mathrm{O}$ ) are transformed in this manner, it is possible to infer that cosmic rays of this energy transverse a gammage $\Sigma_{\mathrm{CR}} \sim 8 \mathrm{~g} \mathrm{~cm}^{-2}$ before escaping the galaxy. This allows us to estimate the total Galactic luminosity in cosmic rays from their observed energy density $U_{\mathrm{cr}}$ and the mean mass of interstellar gas $M_{G} \sim 5 \times 10^{9} M_{\odot}$. This is $L_{\mathrm{CR}} \sim U_{\mathrm{cr}} c M_{G} /$ $\Sigma_{\mathrm{cr}} \sim 3 \times 10^{40} \mathrm{ergs} \mathrm{s}^{-1}$ or roughly three per cent of the average energy input of supernovae into the interstellar medium (at a rate of one per 30 years throughout the Galaxy).

We also measure the energy spectrum of secondaries to have a form $N_{E} \propto E^{-3.3}$ below $\sim 100 \mathrm{GeV}$ in contrast to the primary spectrum $N_{E} \propto E^{-2.7}$. This implies that the cosmic-ray retention time in the Galaxy is energy-dependent $t \propto E^{-0.6}$. This in turn implies that the source spectrum of primary cosmic rays is $\sim S_{E} \propto E^{-2.1}$, in rough agreement with the simple theory of diffusive shock acceleration.

The very highest energy particles with $E \sim 10^{20} \mathrm{eV}$ are also subject to loss as they propagate through the intergalactic medium. The dominant loss is probably photo-pion production on microwave background photons. This limits their acceleration sites of the ones we observe to distances $\$ 100 \mathrm{Mpc}$. This includes many powerful radio galaxies. However, if they are accelerated within the giant double radio components then anisotropy in their angular distribution may also result. Photopion losses, although, do preclude an origin within galaxies. Heavy ions are even more fragile.

High-energy $\gamma$-rays may create electron-positron pairs on microwave background photons. This is relevant to the Whipple observations of $\mathrm{TeV} \gamma$-rays from Mrk421. We would not expect any of the high-redshift blazars to be detected at this energy because the universe should be optically thick at these distances and energies. It is reassuring that no such detection has been reported.

\subsection{Preacceleration}

We have already alluded to the problem of preacceleration for diffusive shock acceleration and impulsive solar flares. In the former case, the major difficulty is associated with electron injection. Basically, we require that particle achieve momenta $p \gtrsim m_{p} a$ before they can scatter off Alfvén waves. For electrons, the associated energy is a factor $\sim m_{p} / m_{e}$ larger than for ions. Whistler modes may help bridge this gap by accelerating electrons above momenta $\sim\left(m_{e} m_{p}\right)^{1 / 2} a$ (Levinson 1992). A fair estimate of the efficiency of whistler preacceleration depends ultimately on numerical simulations of collisionless shocks using hybrid codes.

\section{RECENT DEVELOPMENTS}

Most of the foregoing introductory discussion could have been given (and indeed was given) a decade ago. There have, however, been several changes, mostly brought about by observations, in our view of particle acceleration. These include:

1. It had been proposed that most of high-energy particles observed in terrestrial substorms were created by explosive reconnection in the distant magnetotail. However, direct observation by the AMPTE spacecraft indicates that much of the particle acceleration actually takes place in the near magnetosphere.

2. Earth's magnetosphere was once thought to be a splendid laboratory for diffusive shock acceleration. This may still be the case but the geometrical complications are severe as, typically, the shock changes from quasi-parallel to quasi-perpendicular over its surface. Simulations are proving to be increasingly useful for understanding acceleration under these conditions.

3. The $Y O H K O H X$-ray satellite is bringing about a radical change in our understanding of solar flares. For example, it used to be thought that magnetic loops are filled with gas following the irradiation and evaporation of the chromosphere. It now appears that the soft X-ray emitting gas is always in place at the top of the loop.

4. Our understanding of cosmic-ray acceleration depends critically upon the composition near the "knee" in the spectrum around $\sim 1 \mathrm{PeV}$. The most recent report, from the MACRO collaboration (Ahlen et al. 1992), is that it comprises light particles and not iron as reported earlier. We eagerly await the flight of the GOAL Antarctic balloons, which ought to settle the matter.

5. There may be a big change occurring in our understand of compact X-ray binaries. It is being argued that X-ray novae are black hole systems exhibiting annihilation line and quasiperiodic oscillations (previously thought to be signatures of neutron stars) when in their hard states. It had previously been thought that the $\gamma$-ray continuum from these objects is nonthermal in origin. However, it now appears that Comptonization of self-photons by $\sim 40 \mathrm{keV}$ electrons. (This is still a proper topic for discussion at a conference devoted to particle acceleration because Comptonization is just second-order Fermi acceleration with photons replacing protons and highspeed electrons replacing interstellar clouds!)

6. A somewhat similar change has occurred from $C G R O$ observations of radio-quiet AGNs. Early reports of hard $\gamma$-rays above $\sim 1 \mathrm{MeV}$ now appear to be discounted and there are similarities in their hard X-ray- $\gamma$-ray continua with those of $\mathrm{X}$-ray novae in their hard states. These spectra are not obviously consistent with the much-studied pair plasma models, at least in their simplest forms, in which $\gamma$-rays initiate electronpositron pair cascades. Again, Comptonization by an electronphoton plasma is suggested.

7. In the core-dominated, radio-loud AGNs, it appears from EGRET observations that jets are typically one to two orders of magnitude more luminous in hard $\gamma$-rays than radio to optical emission. The emission mechanism is presumably inverse Compton scattering of soft X-ray photons by $\mathrm{GeV}$ electrons. Particle acceleration in relativistic jets must be both more rapid and more extensive than had previously been thought. This probably has important implications for our understanding of the origin of these jets.

8. Finally, we come to $\gamma$-ray bursters, where the otherwise 
plausible association with local neutron stars is in doubt. If bursters really are at cosmological distances, then, the dynamical and radiative constraints on source models pose the greatest challenge of all to theories of particle acceleration.

All of these issues are controversial. I look forward to lively discussion.
I am grateful to Jon Arons, F. Coroniti, and Hal Zirin for last minute advice and especially to Ian Axford for removing several misconceptions and infelicities from the manuscript. Support under NSF grant AST 89-17765 and NASA grant NAGW 2816 is gratefully acknowledged.

\section{REFERENCES}

Achterberg, A. 1992, in Proc. Graz Predoctoral Astrophysical School, ed. J. van Paradijs, H. M. Maitzen \& J. Heyvaerts (Berlin: Springer), in press

Ahlen, S., et al. 1992, Phys. Rev. D, 46, 895

Arons, J. 1992, in The Magnetospheric Structure and Emission Mechanisms of Radio Pulsars, ed. T. H. Hankins, J. M. Rankin \& J. A. Gil (Zielona Góra: Pedagogical University Press), 56

Arons, J., Gallant, Y. A., Langdon, A. B., \& Yang, T.-Y. B. 1994, in ApJ, Submitted

Axford, W. I. 1992, in Particle Acceleration in Cosmic Plasmas, ed. G. P. Zank \& T. K. Gaisser (New York: AIP), 45

Bastian, T. S., Bookbinder, J., Dulk, G. A., \& Davis, M. 1990, ApJ, 353, 265

Begelman, M. C., Rudak, B., \& Sikora, M. 1990, ApJ, 362, 38

Bell, A. R. 1992, MNRAS, 257, 493

Cameron, R. A., et al. 1993, in Proc. Compton Gamma-Ray Observatory Symp., ed. M. Friedlander, N. Gehrels, D. J. Macomb (New York: AIP), 478

Dawson, J. M. 1989, Sci. Am., 260, 54

Evans, D. S. 1987, in Essays in Space Science, ed. R. Ramaty, T. L. Cline, \& J. F. Ormes (Washington, DC: NASA), 19

Fichtel, C. E. et al. 1993, in Compton Gamma-Ray Observatory Symp., ed. M. Friedlander, N. Gehrels, \& D. J. Macomb (New York: AIP)

Fishman, G., et al. 1993, in Proc. Compton Gamma-Ray Observatory Symp., ed. M. Friedlander, N. Gehrels, \& D. J. Macomb (New York: AIP), 669
Galeev, A. A. 1991, Soviet Phys. JETP, 100, 1811

Goldreich, P., \& Julian, W. H. 1969, ApJ, 157, 869

Hillas, A. M. 1984, ARA\&A, 22, 425

Jones, F. C., \& Ellison, D. C. 1991, Space Sci. Rev., 58, 259

Kuijpers, J. 1990, in Proc. IAU Symp. No. 142, Basic Plasma Processes on the Sun ed. E. R. Priest \& V. Krishan (Dordrecht: Kluwer), 365

Lee, M. A. 1992, in Particle Acceleration in Cosmic Plasmas, ed. G. P. Zank \& T. K. Gaisser (New York: AIP), 27

Levinson, A. 1992, in ApJ, 401, 73

Mattox, J. R., 1992, BAAS, 24, 1296

Meegan, C. A., et al. 1992, Nature, 355, 143

Melrose, D. B. 1992, in Particle Acceleration in Cosmic Plasmas, ed. G. P. Zank \& T. K. Gaisser (New York: AIP), 3

Parks, G. K. 1991, Physics of Space Plasmas (New York: Addison Wesley) Punch, C. W., et al. 1992, Nature, 358, 477

Reynolds, S. P., \& Ellison, D. C. 1993, ApJ, 399, L75

Ruderman, M. 1991, in Particle Acceleration near Accreting Compact Objects, ed. J. van Paradijs, M. van der Klis \& A. Achterberg (Amsterdam: Royal Netherlands Academy of Arts \& Science), 69

Sunyaev, R. A., et al. 1992, ApJ, 389, L75

Van Allen, J. A. 1987, in Essays in Space Science, ed. R. Ramaty, T. L. Cline, \& J. F. Ormes (Washington: NASA), 1 